Lina Ojeda Revah*

GuAdalupe Álvarez*

\title{
LA REFORESTACIÓN DE TIJUANA, BAJA CALIFORNIA COMO UN MECANISMO DE REDUCCIÓN DE RIESGOS NATURALES
}

\begin{abstract}
RESU MEN
El medio físico en el que se asienta la ciudad de Tijuana presenta ciertas características que hacen muy compleja su problemática ambiental. Es una zona vulnerablea inundaciones, en la que por su formación geológica existen derrumbes potenciales, los cuales aumentan debido a que también es una zona sensible desde el punto de vista sísmico. Asimismo, la vegetación que rodea a la ciudad ha evolucionado adaptándose a incendios naturales frecuentes por lo que requiere de ellos para su permanencia. El estilo de desarrollo urbano al igual que el concepto restringido de lo que constituyen las áreas verdes de Tijuana han acrecentado el grado de riesgo a este tipo de perturbaciones naturales. De hecho, según el XIV Ayuntamiento de Tijuana, $40 \%$ de la población de la ciudad vive en zonas de alto riesgo. Con base en lo anterior se propone una estrategia de forestación urbana, orientada especialmente hacia las laderas, creando una red de áreas verdes, con la finalidad de disminuir los riesgos a los que la población está expuesta por inundaciones, deslaves y derrumbes, a la vez quesemantengan los flujos naturales y se reduzca el actual déficit deáreas verdes.
\end{abstract}

\section{A BSTRACT}

The physical setting the city of Tijuana is currently settled on displays certain characteristics that make its environmental set of problems quite a complex one. This is azonethat is vulnerableto floodings. Dueto its geological formation potential landslides are ever present and ever increasing since, from the seismic stand point, this is a critical zone as well. Furthermore, the city's surrounding vegetation has evolved in such a way that it has adapted to frequent natural fires; it thus needs such fires for its permanence. The urban style of development as well as the the limited notion of green areas in Tijuana has brought about an increasein the degree of risk to such types of natural disturbances. In fact, according to Tijuana's XIV Municipal Authorities (A yuntamiento), 40\% of the city's population lives in high risk zones. Due to the aforementioned, a strategy for urban forestation mainly oriented towards the hillsides is proposed. The aim is to create a network of green areas so as to diminish the risks the population may be exposed to on account of floodings, landslides or landslips, whilesimultaneously preserving thenatural flows and reducing the current deficit of green areas.

\footnotetext{
* Departamento de estudios urbanos y del medio ambiente. El Colegio de la Frontera NORTE. Correo electrónico: Iojeda@mail.colef.mx
} 


\section{INTRODUCCIÓN}

Debido a las características del medio físico en el que se desarrolla, la ciudad de Tijuana es susceptible a fenómenos o perturbaciones naturales queindependientementedelas acciones que sereal icen para su control, siempre volverán a presentarse.

Esta es una zona al tamente vulnerable a inundaciones, en la que por su formación geomorfológica existen derrumbes potenciales, los cuales aumentan debido a que también es una zona sensi ble desdeel punto de vista sísmico. Asimismo, la vegetación querodea a la ciudad ha evolucionado adaptándose a incendios natural es frecuentes por lo que requiere deellos para su permanencia.

Sobre este tipo de fenómenos, la teoría ecológica ha modificado su visión de equilibrio, al reconocimiento dela existencia de procesos de perturbaciones ambientales, incertidumbrey riesgo que provocan que los ecosistemas cambien continuamentesobreciertos rangos quevarían con el tiempo y la escala (Wolch, 1996). Se considera perturbación al evento que altera significativamente el patrón de variación de la estructura y función de un sistema.

En la ciudad de Tijuana, nuestra área de estudio, las inundaciones y el control de incendios naturales constituyen perturbaciones que destacan por sus implicaciones en el aumento de los riesgos a los que está expuesta la población de esta ciudad. Sin embargo, hasta la fecha estas perturbaciones naturales tan solo han sido consideradas como accidentes azarosos y no como los eventos recurrentes que son. Por ello, las perturbaciones natural es deberían ser consideradas dentro de los planes dedesarrollo urbano, diseñando mecanismos quemitiguen su impacto.

Con base en lo anteriormente expuesto, se propone una estrategia de forestación urbana, que consiste en crear una red de áreas verdes, orientada especial mente hacia las laderas y cañones, con la finalidad de disminuir los riesgos existentes dentro de la ciudad por inundaciones, deslaves y derrumbes principalmente, a la vez que se mantengan losflujos naturales y se reduzca el actual déficit deáreas verdes en la ciudad. 


\section{MATERIAL Y MÉTODOS}

Con baseen fotos aéreas a col or dela ciudad deTijuana, escala 1:45000 tomadas por la A dministración Nacional del Océano y la A tmósfera (NOAA) delosEstados Unidos en agosto de 1994y usando como Sistema de Información Geográfica el SAVANE, desarrollado por el Institut Scientifiquepour leDéveloppement en Coopération (ORSTOM) seelaboró un mapa en el quesemuestran lasáreas verdes existentes en la ciudad, incluyendo aquel las que no tienen un uso recreativo y se determinan con base en las pendientes, las áreas susceptibles de ser forestadas o conservadas.

Con la final idad deconocer el concepto deárea verdequesemaneja dentro del municipio y los criterios usados para su establecimiento, diseño y mantenimiento, se realizaron 20 entrevistas con funcionarios públicos relacionados con la creación de áreas verdes y disminución de riesgos dentro de la ciudad (Direcciones de Obras y Servicios Públicosy dePlaneación y Ecología Urbana, Delegaciones Municipales y Parque Morelos). Aunado a lo anterior, se realizó una evaluación general dela adecuación de las especies usadas por el municipio para reforestar.

\section{DESCRIPCIÓN DEL ÁREA}

La ciudad de Tijuana está situada dentro de la región biogeográfica denominada Región Californiana. Esta región, ubicada del lado del Pacífico se extiende desde la costa hasta los 2600 msnm, y desde la frontera con los Estados Unidos, hasta el margen sur del Llano Santa María (Wiggins, 1980).

La Región Californiana presenta una topografía accidentada, un climatipo mediterráneo, esto es, seco y templado, con lluvias invernales de 270 mm anuales en promedio y cálido extremosos (García y Mosiño, 1968). En ella existe una gran variedad de suelos que contienen desde poca hasta mucha materia orgánica, con texturas desdearenosas hasta arcillosas y de color amarillento hasta rojizo (SPP, 1984).

Geomorfológicamente, el área urbana de Tijuana está constituida por al uviones recientes, terrazas fluviales y marinas, por formaciones 
derocas cáusticas y sedimentarias y, finalmente, por rocas denaturaleza volcánica. A simismo existen por lo menos tres direcciones por sistemas de fallas geológicas muy notorias. Todos estos elementos dan como resultado movimientos de ladera; de ellos los más comunes son las fluencias de detritos, deslizamientos rotacionales y caída de bloques (García, 1988).

Los tipos devegetación característicos delos al rededores deTijuana son el matorral costero, el chaparral, la vegetación riparia y el pastizal . El primero es un matorral parcialmente caducifolio, ${ }^{1}$ de menos de $1 \mathrm{~m}$ de altura. En este tipo de vegetación destacan especies como la "al canforilla" (A rtemisia californica), "valeriana" (Eriogonum fasciculatum), "flor de mayo" (V iguiera laciniata) y "hierba del vaso" (Encelia californica) (Rzedowski, 1978).

El chaparral es una asociación dearbustos perennifolios ${ }^{2}$ quemiden aproximadamente de $5 \mathrm{~cm}$ a $3 \mathrm{~m}$ de altura. Las especies más comunes son A denostoma fasciculatum en la costa, y la "hierba del pasmo" (A denostoma sparsifolium) y algunas especies de "manzanitas" (A rctostaphylos spp.) tierra adentro. Otros componentes comunes son "ramón" (Cercocarpus spp.), "toyón” (H eteromeles arbutifolia) y “yuca” (Y ucca whipplei) (Rzedowski, 1978).

La vegetación riparia se caracteriza por la presencia de Platanus racemosa (aliso) y M alosma laurina (lentisco), la primera arbórea y la segunda arbustiva. En su modalidad arbustiva la vegetación riparia presenta especies como R hamnus crocea y R hus integrifolia.

Por último, los pastizales se componen de anuales de invierno provenientes en su mayoría de las tierras que rodean al Mar Mediterráneo. Incluyen a Bromus rubens, B. driandus, A vena fatua, A . barbata, Erodium circutarium, Brassica geniculata, y algunas nativas como H ermizonia spp. (M innich y Franco, 1998). Generalmente forman una capa herbácea continua de 0.5 a 1.5 metros dealtura (M innich, 1983).

${ }^{1}$ Especies que tiran las hojas durante la temporada seca.

${ }^{2}$ Las especies perennifolias son aquellas que mantienen su follaje durante todo el año. 
Sobrela vegetación, cabe destacar queel matorral costero es un tipo de vegetación único en el país, que sostiene un elevado número de endemismos y queha visto dismi nuida deforma al armantesu área de distribución natural a causa de cambios en el uso del suelo, especial mentepara ocupación urbana.

\section{INUNDACIONES}

Las grandes inundaciones queexceden el área normalmenteabarcada por las planicies inundables pueden ser consideradas perturbaciones. Por su topografía accidentada y precipitaciones intensas de poca duración que se traducen en escurrimientos rápidos, la ciudad de Tijuana es muy vulnerable a este tipo de eventos.

Según un estudio realizado por Bocco, et. al. (1993) aunque en Tijuana el periodo deretorno degrandesinundaciones es bajo (75años), éste puede repetirse en promedio, cada tres años, con 0.7 de probabilidad.

Los problemas que provocan las inundaciones seagravan debido a que dentro de la ciudad existen áreas con formaciones geológicas de sedimentos marinos que presentan derrumbes potenciales y suelos arcillosos grises que se expanden cuando son humedecidos por las Iluvias. Otras áreas suscepti bles a derrumbes tienen suel os arcillosos, topografía levemente ondulada y vegetación herbácea (Oberbauer, 1986). La mayoría de estos deslizamientos y derrumbes comúnmente son disparados o reactivados durante la temporada de lluvias. Debe considerarseademás queen el área ocupada por la ciudad existemicrosismicidad asociada a fallas geológicas que provoca deslizamientos y movimientos del terreno en varios sectores de la ciudad (A ragónArreola, 1994).

En 1993 a raíz de una gran inundación resultaron dañadas 1960 hectáreas, esto es, $10 \%$ del área urbana. De ésta, 57\% fue dañado por erosión-remoción en masa (en cañonesy laderas), 20\% por depositación (especialmenteen lasterrazas bajas, a lo largo del cauce del Río Tijuana) y el 23 restante por anegamiento (superficies cumbrales) (Bocco, et. al., 1993). 
Con base en datos de geomorfología y de pendientes, actual mente se sabe que casi un $15 \%$ del área ocupada por la ciudad es susceptible al proceso de erosión-remoción en masa y que éste se distribuye en casi toda la zona urbana principal menteen cañadas, Iomeríos y cerros (Romo, 1996).

\section{INCENDIOS}

Los incendios naturales periódicos son considerados perturbaciones en ecosistemas que están adaptados a ellos dada su frecuencia, esto es, la persistencia desus especies dependedelos incendios. Sin embargo la prevención de los mismos también sería considerada una perturbación ya quelas especies presentes sucumbirían a las invasiones externas (Forman, 1995). En el caso deTijuana, los tipos devegetación que rodean a la ciudad, esto es, el chaparral y el matorral costero, han evolucionado para adaptarseal fuego.

Durante el presente siglo, debido a la preocupación por el manejo decuencas hidrológicas y daños depropiedad, seestablecieron diversas políticas de manejo de incendios en el sur de California. Hasta hace pocos años, la estrategia más común era la supresión organizada de incendios.

Estudios comparativos sobre los efectos que tienen los diferentes manejos de los incendios naturales en California y Baja California, indican que la supresión de incendios realizada en Estados Unidos tieneun impacto mínimo en el matorral costero y en los pastizales. Sin embargo, el control de incendios sobre el chaparral reduceel número de incendios provocando que éstos aumenten de tamaño, capacidad de dispersión e intensidad Ilegando a ser incontrolables bajo condiciones de clima severo. Deesta forma los incendios quesedan al sur de la frontera son más numerosos, frecuentes y pequeños quelos que se dan en California (Minnich, 1983 y 1996).

Basándose en este tipo de estudios, se han implementado recientemente en el sur de California, programas de prescripción de incendios como una estrategia para lograr entre otros objetivos, la reducción de combustible. Sin embargo, éstos se han realizado sin un entendimiento claro de sus efectos ecológicos a largo plazo. 
Actual mente, la frecuencia promedio de incendios en el chaparral es de entre 20 y 40 años, aunque esta cifra pudo haber sido menor antes de los asentamientos europeos. En el matorral costero, su frecuencia natural es probablementemás cercana al límiteinferior antes mencionado, dada la menor incidencia de rayos en las áreas costeras menos elevadas. A unque los rayos son considerados la fuente natural de incendios, pero en las últimas décadas su ocurrencia ha sido en gran parte ocasionada por el hombre (O'Leary, 1989).

En el caso particular de Tijuana, en parte debido al crecimiento desordenado y por la falta de regulación y vigilancia en las construcciones, la vegetación de los al rededores dela ciudad presenta un severo deterioro y por lo tanto el riesgo por incendio no es al to.

\section{DESARROLLOURBANO}

En estemedio físico y bajo esterégimen de perturbaciones, Tijuana ha experimentado un desmedido crecimiento urbano, especialmente durante las últimas décadas. Tanto su extensión, como la modalidad de su desarrollo, han generado una serie de problemas que no sólo afectan a los ecosistemas quela rodean, sino que éstos se revierten a la propia ciudad, al aumentar significativamente el grado de riesgo al queseexponela población debido a inundaciones, deslaves, derrumbes y movimientos sísmicos.

En lo que se refiere a su extensión, tan sólo en 20 años la superficie queocupala ciudad setriplicó sobreáreas originalmentecubiertas por zonas agrícolas, vegetación riparia y matorral costero, un tipo de vegetación único en el país. Sin embargo, la ciudad se asienta en una zona con condiciones fisiográficas difíciles y aunque su crecimiento originalmente se llevó a cabo sobre suelos aptos para uso urbano, conforme ha crecido se han ocupado espacios con pendientes más acusadas. Actualmentetres cuartas partes dela ciudad selocalizan en laderas con una inclinación de menos de $15 \%$, pero casi un $8 \%$ se localizan en laderas con pendientes de más de $25 \%$ deinclinación (ver figura 1). Dado que existe poca superficie urbanizable a bajo costo, es la población de bajos recursos la queocupa laderas muy empinadas y fondos de valle expuestos a deslaves e inundaciones. 
En Tijuana la apropiación del espacio urbano se realiza principal mente de tres formas: con asentamientos irregulares, con la creación defraccionamientos habitacionalesy con parquesindustriales.

La mayor parte de los asentamientos irregulares seexpanden sobre ejidos, laderas con pendientes muy acusadas o en el fondo de los cañones. En ellos las construcciones realizadas, debido a los escasos recursos con los que se cuenta, se realizan creando terrazas con Ilantas de desecho, quedebido a la forma en que son utilizadas pueden llegar a constituir estructuras muy frágiles para impedir posibles derrumbes.

En el caso de los fraccionamientos y parques industriales, éstos por lo general cuentan con presupuesto suficiente para la compra de terrenos planos o en su defecto para el aplanado de los mismos. En este proceso generalmente se arroja el exceso de tierra a las laderas colindantes. Por la falta de regulación y control de las construcciones, las laderas en particular y los terrenos en general, son desprovistos totalmente de cubierta vegetal. Ambas formas de apropiación del espacio se desarrollan sin consideración del riesgo que puedellegar a implicar la eliminación de la vegetación natural.

Sobre esto, actualmente se sabe que la pérdida de vegetación, incrementa los escurrimientos deagua y el depósito desedimentos en los canales colectores, decreciendo así su capacidad de transporte. El proceso de urbanización incrementa la magnitud y la frecuencia delas inundaciones, al aumentar las superficies altamente impermeables, impidiendo la infiltración de agua y ocasionando escurrimientos que llegan a saturar los drenajes (Smith, 1992), especial mente durante la época de lluvias. Deigual forma la vegetación natural juega un papel muy importanteen la estabilización deladeras, por lo quesu remoción aumentalos riesgos dedeslaves. Cabemencionar además queel proceso deerosión intensa puedeaumentar los riesgos a la salud, al el evarsela cantidad de partículas suspendidas en la atmósfera.

Aún más, en las zonas urbanas, el al terar o suprimir la vegetación y no sustituirla, acarrea consigo muchos problemas ecológicos. Al carecer de una capa vegetal, el microclima puede ser alterado ya que la vegetación actúa como un agenteestabilizador. Sin vegetación, el suelo es susceptibleala erosión y al propiciarseel escurrimiento deagua, se dificulta la filtración de la misma y por tanto la recarga de los mantos 
acuíferos. En un medio urbano, con altos niveles de emisiones de monóxido de carbono, la carencia de una capa vegetal no permite la oxigenación del área ni la filtración de contaminantes, propiciándose así un ambiente contaminado. Asimismo, la desarticulada presencia devegetación, junto con la dominancia deelementosartificiales, genera problemas de deshumanización de los espacios por la frialdad de los materiales de construcción y su poco atractivo visual (Bazant, 1988).

En Tijuana, la denudación de las laderas, junto con el rezago existente en la construcción de infraestructura (como la falta de pavimentación) aumentan la cantidad de sedimentos acarreados a las partes bajas del valle.

\section{ÁrEASVERDESEN TIJUANA}

En una ciudad como Tijuana, en donde el acelerado crecimiento dela población ha sobrepasado la capacidad de construir infraestructura básica, no sorprende la carencia de parques y jardines o áreas verdes en general. Sin embargo, esta real idad es mucho más al armante. Desde un punto de vista estrictamente espacial, en 1994, Tijuana poseía al rededor de $4.16 \mathrm{~m}^{2}$ de área verde por habitante (figura 2, cuadro 1). Considerando solamente su utilidad social, esto es, los parques y jardines que pueden ser usados por la población en general, la cifra disminuyea $1.3 \mathrm{~m}^{2}$ por habitante. Lo importantea destacar es queambas cifras se encuentran muy por debajo de las normas dictadas internacional mente, que recomiendan entre 10 y $12 \mathrm{~m}^{2}$ por habitante.

A este punto habría que añadir que no todas las Ilamadas "áreas verdes" consideradas en eseíndiceson verdaderamenteáreas cubiertas por una capa vegetal, sino que simplemente han sido bautizadas o designadas como parques. La cobertura vegetal que muchos de estos tienen, oscila entre 20 y $30 \%$ del área considerada como parque e inclusiveen otros, es completamenteinexistente.

La determinación del área destinada a la creación de parques y jardines en Tijuana tiene sus raíces en el Reglamento de Fraccionamientos del Estado de Baja California. En dicho reglamento, en el Capítulo II, artículos 32 al 43, se establece que para cumplir con las necesidades de parques y jardines, los fraccionamientos “deberán 
destinar una superficie que no sea menor al de un 3\% de la superficie vendible. Los parques y jardines serán construidos por el fraccionador y seobligaráa conservarlos y mantenerlos por su cuenta hasta en tanto el fraccionamiento no sereciba por el Gobierno del Estado. El Ayuntamiento del Municipio quesetratecontinuarásu atención en el momento en el quelo reciba de parte del Gobierno del Estado".

Independientemente de que ese $3 \%$ para establecer parques y jardines es un porcentajemuy bajo, el del imitar dichas áreas en función de un porcentaje de superficie urbanizada es criticable, debido principal mentea las diferencias de densidad de población quese dan en las zonas habitacionales. Idóneamente, la delimitación de áreas verdes en una zona urbana debiera ser en función del área foliar ${ }^{3}$ por cada habitante (Barradas y Seres, 1988). Sin embargo, este criterio resulta poco práctico en las zonas áridas o semiáridas, en donde la vegetación natural está más esparcida y el mantenimiento de mucha vegetación es casi imposibledada la escasez del recurso agua. Dehecho, la mayoría de los parques deTijuana carecen de vegetación por la fal ta deabastecimiento del líquido. Pocos son losquereciben agua por medio de pipas y un número todavía menor es el quecuenta con un sistema deriego.

Para forestar los parques y avenidas de la ciudad, se utilizan pocas especies que son muy tolerantes a las condiciones urbanas y cuyo crecimiento rápido está casi asegurado, pues se conocen bien sus necesidades de cultivo. La mayoría de estas plantas proceden de regionesmuy diferentes. Además, existeuna gran tendencia a depender cada vez más de plantas ornamentales muy manipuladas genéticamente, que aunque muy conocidas y vistosas, pertenecen a un número de especies muy reducido. Desafortunadamente, es poco lo queactual mentesehace por incrementar el número deplantas deornato a partir dela flora local, a pesar de que existe un enorme potencial.

${ }^{3}$ Área que suman las superficies de las hojas de una planta. 
FIgURA 1. Áreas verdes de la ciudad de Tijuana, B.C. 1996.

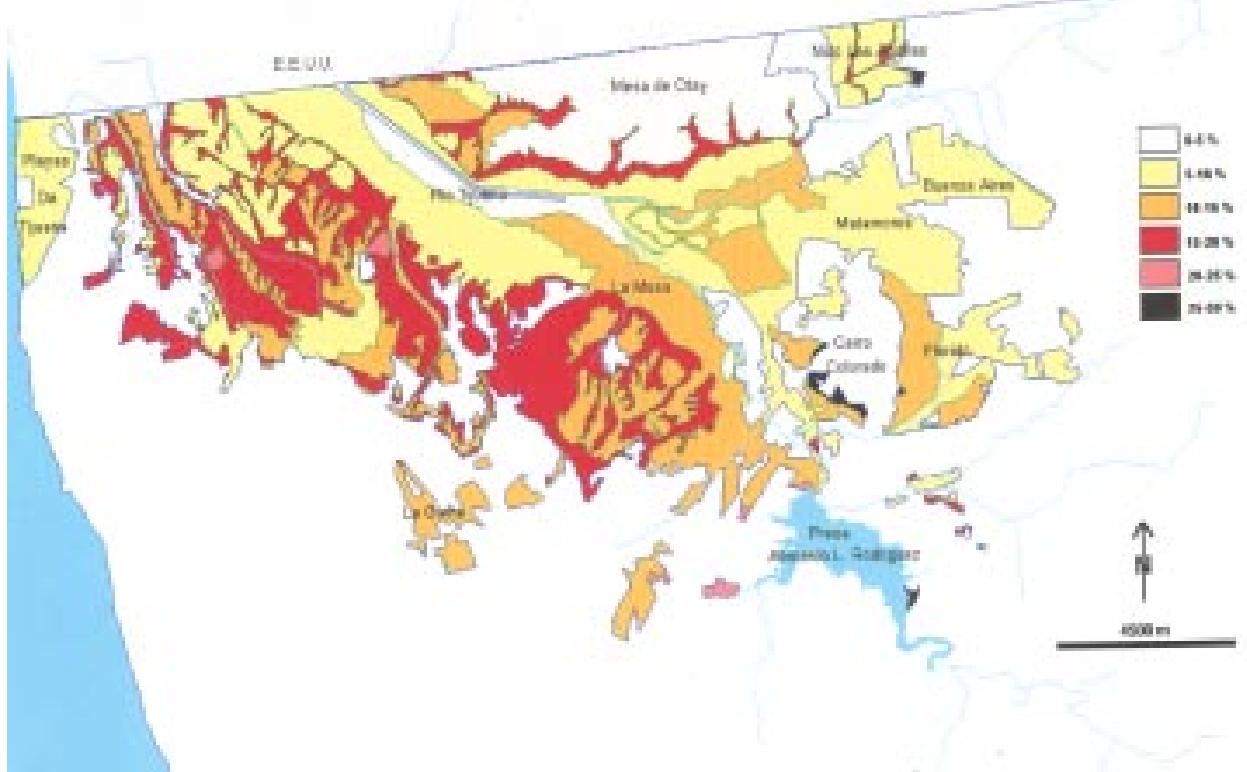

Fuente: Elaborado por Guadal upe Alvarez. Maestría en Administración Integral del Medio Ambiente. CICESE-El Colef-oRSTOM. 
FIGURA 2. Rangos de pendientes por conjuntos fisiográficos.

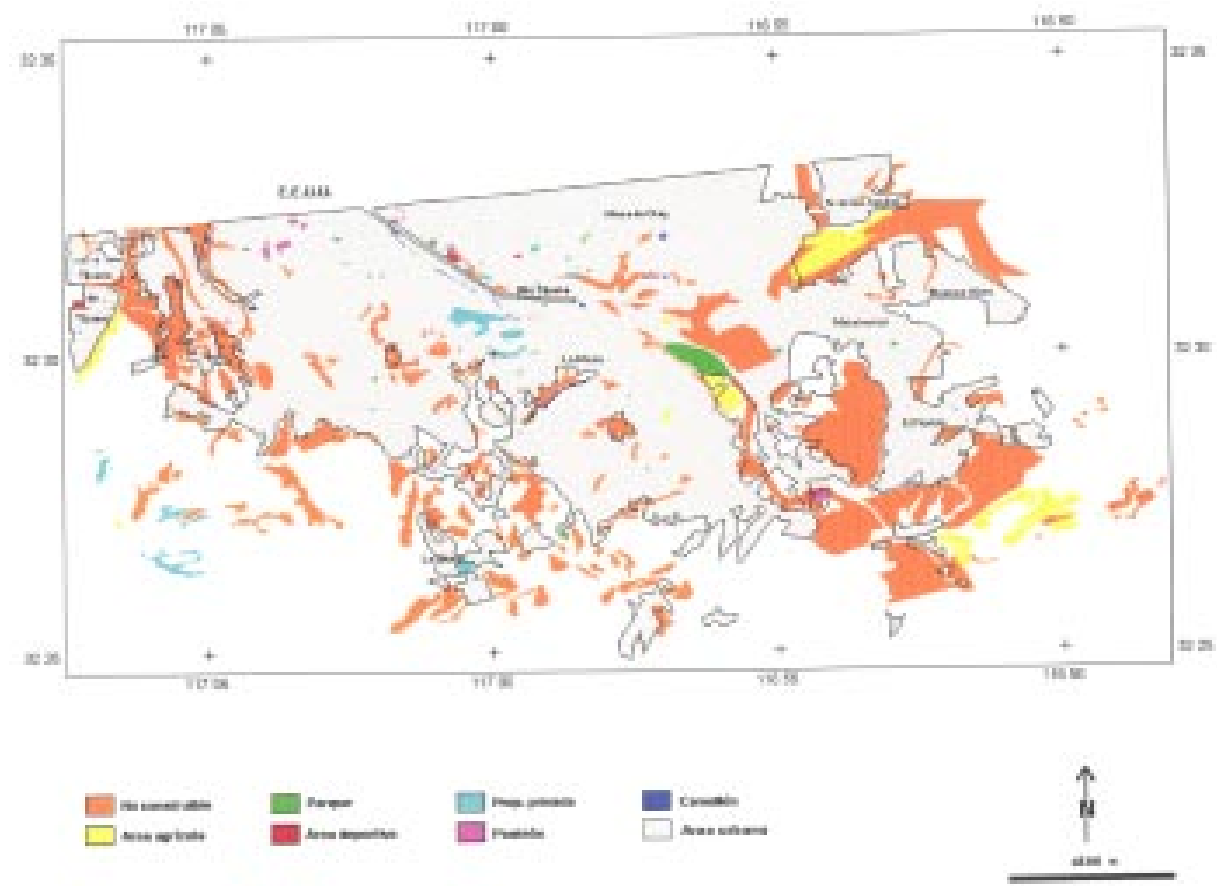

FunNte: SIgEF, Proyecto ORSTM-COLEF. 
CUADRO 1. Áreas verdes de la ciudad de Tijuana (1994).

\begin{tabular}{|lr|r|}
\hline Área verde & Unidades & $\mathrm{m}^{2}$ \\
\hline Camellones & 116 & 400152 \\
Parques & 45 & 1225323 \\
Áreas deportivas & 10 & 20055 \\
Subtotal & 171 & 1645530 \\
Propiedad privada & 28 & 1950191 \\
Panteones & 7 & 345890 \\
Total & 206 & 3941611 \\
& & \\
\hline
\end{tabular}

Fuente: Álvarez, M.G. (1996:98).

Pero el panorama es aún más complejo, Tijuana está situada en una región semiárida. Siendo uno de los factores más limitados el recurso agua, generalmente se hace uso de grandes cantidades de la misma para crear áreas verdes. De las 35 especies estudiadas, que son utilizadas por el Municipio deTijuana, sedeterminó que $20 \%$ requieren de riego diario, $49 \%$ de dos a tres veces por semana y tan sólo $31 \%$ son resistentes a la sequía, esto es, requieren riego ocasionalmente. En lo quese refierea su contribución para mitigar los problemas ambientales principales a nivel local, se encontró que sólo el 9\% de ellas controlan la erosión por agua y un $17 \%$ por viento. Por último, debemencionarse que $51 \%$ de las especies usadas son arbóreas y el 34\% son arbustivas.

Delas cifras anteriores se deduce que no existeuna estrategia en la selección de especies a utilizar de acuerdo con las necesidades ecológicas y a la problemática de riesgos a la que está sujeta la ciudad deTijuana.

PARTICIPACIÓN CIUDADANA, ADMINISTRACIÓN PÚBLICA Y ÁREASVERDES

Dentro del municipio de Tijuana las dependencias encargadas de la creación, diseño, establecimiento de áreas verdes y participación ciudadana son la Dirección de Obras y Servicios Públicos 
(Departamento de Parques y Jardines y Viveros); la Dirección de Planeación y Ecología Urbana (Departamentos de Fraccionamientos, de Planeación y Proyectos y de Desarrollo Social); las Delegaciones Municipales y el ParqueM orelos.

En las entrevistas realizadas a diferentes funcionarios pertenecientes a estas dependencias destaca princi palmentela concepción restringida que se maneja de lo que constituyen las áreas verdes urbanas, la cual en todos los casos se limitó a los beneficios deoxigenación, recreación y ornato que se derivan de ellas. Asimismo, quedó de manifiesto la desarticulación existente entre la mayoría de los Departamentos y Direcciones involucradas en la forestación urbana y la creación y mantenimiento de áreas verdes. No hay por ejemplo comunicación directa entre el Departamento de Parques y Jardines y el programa abocado a la prevención de riesgos. Consecuentemente, es lógico esperar quenunca haya existido una estrategia integral a nivel detoda la ciudad para el establecimiento deáreas verdes dentro de los planes de desarrollo urbano. Las áreas verdes se establecen por lo general en función de la demanda de la población, la cual no ha sido informada deello.

En resumen, la limitada y desarticulada concepción de las áreas verdes urbanas dentro del propio municipio, quees compartida por la población en general, se refleja en el número, extensión, ubicación, diseño y grado ded eterioro que presentan las áreas verdes deTijuana.

Sin embargo, antela mayor difusión delos problemas ambientales, la comunidad ha comenzado a mostrar mayor interés por el ambiente y como respuesta el municipio ha iniciado una serie de acciones, que desgraciadamente siguen siendo muy puntuales, aisladas y bajo una concepción muy restringida.

La educación ambiental seestá implementando en las escuelas y el ayuntamiento ha planteado programas de forestación con la participación ciudadana. Desgraciadamente esta participación es aún incipientey sól o se concreta a sembrar árboles queson proporcionados por el propio ayuntamiento. Los programas de forestación que se realizan aún no toman en cuenta los aspectos ecológicos regionales importantes para restablecer los cicl os biogeoquímicos al terados por la expansión urbana y servir demedidas atenuantes contra los riesgos 
naturales. Normalmente se donan ciertos árboles a la población entusiasta que quiere colaborar para "ayudar a la ecología” deTijuana y en la gran mayoría de los casos, no seconsideran ni las características particulares delas especies usadas, ni las condiciones ecológicas en las quese van a plantar, ni se tiene un seguimiento de su supervivencia.

Dentro de este panorama nada al entador, un acierto recientemente implementado para el mantenimiento delasáreas verdesesel programa "adopta un parque" promovido por el municipioy dirigido a lasempresas o compañías privadas. Con un enfoqueun poco más integrado y a raíz del problema generado por el proyecto de desarrollo urbano que disminuyó el área originalmente otorgada al único parque metropolitano deTijuana: el Parque Morelos, el municipio de Tijuana creó en 1997 el Comité deForestación del Municipio.

En lo queserefierea la participación ciudadana actualmenteexisten al gunas organizaciones no gubernamentales interesadas en la creación y mantenimiento de las áreas verdes urbanas, entre las que destacan el "Patronato parala defensa, recuperación y preservación delas áreas verdes del fraccionamiento de Playas de Tijuana, y el Movimiento Ecologista deBajaCalifornia (MEBAC).

\section{FORESTACIÓN EN TIJUANA}

En el caso particular de Tijuana, se requiere de una estrategia de forestación que mitigue el impacto ambiental negativo que le ha causado su acel erado crecimiento. Un programa deesta naturaleza debe considerar las necesidades queen materia deecología urbana demanda la región, incluyendo aquel las perturbaciones natural es a las que está sometida inevitablemente. Debe responder a varias condiciones: su ubicación dentro de una zona semiárida, con topografía muy accidentada, con riesgo de inundaciones, derrumbes, deslizamientos y con microsismicidad, que sostiene una flora muy diversa, peculiar, adaptada a incendios y con un número de especies endémicas muy elevado que debe ser conservada; y por último, la posibilidad de aprovechar la existencia de grandes cantidades de agua para usos urbanos, traída en forma artificial, pero desperdiciada y manejada en formairracional. 
Para ello conviene diferenciar dos conceptos. Estos dos términos son "parquesy jardines" y "áreas verdes", que pueden ser manejados con estrategias diferentes de forestación de acuerdo con su ubicación y su uso. El tradicional término "parques y jardines" se refiere exclusivamente a aquellos sitios que sean de uso recreativo. Se trata de centrar estas áreas a actividades humanas propias de una ciudad. Los parques y jardines, dada su naturaleza basada en amplias extensiones, generalmente planas, cubiertas de pasto y árboles frondosos, requieren de grandes cantidades de agua para su mantenimiento. Su vegetación normalmente está compuesta por especies propias de regiones donde la precipitación pluvial es superior a la Región Californiana.

La concepción de lo que constituye la vegetación urbana se puede ampliar introduciendo el segundo concepto: el de "áreas verdes". En ellas quedarían incluidos aquellos terrenos en donde a causa de las condiciones naturales del sitio, resulte imposible realizar construcciones, como podrían ser pendientes muy pronunciadas, cañones muy cerrados, etcétera. El objetivo principal deestas áreas sería el controlar la erosión y mitigar los efectos causados por las inundaciones. Sin embargo, también contribuirían a mejorar las condiciones del medio ambiente y podrían ser usadas como áreas de recreación.

El enfoquede dichas áreas podría ser llamado ecológico, ya queen ellas seles permitiría a los elementos funcionar en una forma natural . Podrían variar mucho en tamaño, tenderían a ser informales, exhibirían varios grados de carácter local, requerirían un mínimo de gasto en diseño o no lo requerirían, y uno de sus mayores atractivos sería el bajo nivel de manejo requerido, ya que se permitiría que tome curso una sucesión vegetal, introduciendo un el emento dinámico. La presencia de vida silvestre sería un atractivo, las orillas abruptas serían reemplazadas por ecotonos, ${ }^{4}$ dominarían las especies nativas y las

${ }^{4}$ A sociación vegetal de transición producida, entre otras dos, por invasión mutua (Font Quer, P. 1975:354). 
natural izadas y sobretodo sería un hábitat para quela genteinteractúe, ya que estaría compuesto por las especies mejor adaptadas al sitio, capaces de perpetuarse solas (Gil bert, 1989).

Dentro del manejo de estas áreas es necesario considerar que parte dela vegetación natural está naturalmente adaptada al fuego y requiere deél para sobrevivir. Ello quieredecir queno sepodría permitir queel material muerto seacumuleo quesemanejela composición deespecies. El material muerto podría ser usado para la elaboración decomposta o como combustiblecontrolado.

Las áreas verdes "ecológicas" podrían tener una gran gama de modalidades. Una consistiría en apartar dentro de las zonas urbanas fragmentos de tierra sin perturbar, esto es, con la vegetación natural ya existente en ellos. Otra modalidad consideraría el hacer uso de la vegetación nativa en zonas al tamente perturbadas para crear áreas verdes. Una tercera modalidad incluiría aquellos espacios en los que simplemente se permitiera un proceso de sucesión vegetal natural, aprovechando el banco desemillas ya existenteen el área, como lo que sucede en muchos terrenos baldíos abandonados por largo tiempo. Cabe mencionar que en todas estas modal idades dada su naturaleza (dentro de una zona urbana) con mucha influencia de semillas provenientes de áreas verdes cultivadas en sus al rededores, sería rico en plantas exóticas.

Considerando la escasez de agua las áreas verdes ecológicas conservarían paisajes naturales dezonas semiáridas, también conocidas como paisajes xerófitos. ${ }^{5}$ Esto es, se mantendrían paisajes naturales y jardines con plantas adaptadas a sequías y por lo tanto con el uso de muy poca agua. El paisajismo xerófito trata de adaptarse al medio ambienteárido que lo rodea, más queintentar trasplantar y mantener paisajes con especies provenientes de zonas más húmedas, que consumen mucha agua.

${ }^{5}$ Xerófito: derivado del griego xeros, que significa seco. 
Los ecosistemas naturales que rodean a la ciudad de Tijuana, precisamente por desarrollarse en un clima semiárido pueden ser manejados como paisajes xerófitos. En ellos habitan muchas plantas cuyas características morfológicas les confieren un gran potencial ornamental. Estas plantas silvestres ofrecen soluciones a muchos problemas ya que, a diferencia de las plantas domesticadas, tienen la habilidad de crecer solas sin la ayuda del hombre.

Un programa de forestación que estableciera una red de este tipo de áreas verdes podría resultar de gran beneficio para la ciudad de Tijuana. Con la existencia de vegetación se reduciría la erosión, fenómeno que produce pérdida de grandes cantidades de suel o fértil, la necesidad derecolectar y transportar sedimento depositado por las Iluvias en zonas pavimentadas, y el deterioro que producen las corrientes pluviales sobre viviendas y vialidades. Asimismo, se contribuiría mantener un climamás estableen la ciudad y un ambiente más sano. Los beneficios hidrológicos y deregulación deinundaciones dela cubierta vegetal serían: mitigación de los daños ocasionados por inundación, recarga de los mantos acuíferos y mayor seguridad en el abastecimiento deagua.

Dentro de la ciudad existen 50734 hectáreas de superficie con pendientes mayores al 30\% de inclinación, compuestas por cañadas, zonas de escurrimiento, lechos de río en los que no es recomendable la urbanización y en dondees probablequeexistan relictos devegetación nativa (figuras 1 y 2). El transformar estas zonas en áreas verdes podría elevar el índice deárea verde por habitante.

\section{DISCUSIÓN Y CONCLUSIONES}

La clave del deterioro del medio natural estriba en la aceleración de flujos y ciclos que tanto el ambiente natural y en menor medida, el rural tienden a mantener lentos. La puesta en marcha de procesos graves de erosión mecánica y la pérdida de riqueza y diversidad biológica y de pautas del paisaje son claros ejemplos (Díaz Pineda y Valenzuela, 1989).

Desgraciadamentela búsqueda de nuevas opciones de urbanización no seconsideran sino hasta que se presenta una perturbación o evento 
desastroso con los al tos costos social es que representan y cuando sus efectos son irreversibles.

En el caso de Tijuana, a nivel regional, el agua y la sismicidad han jugado el papel de disparadores de deslizamientos; a nivel local la modificación del talud por actividad humana es un agenteimportante, en gran parte por los masivos movimientos de tierra realizados sin control en las partes altas para la construcción de nuevos fraccionamientos residenciales o parques industriales, la urbanización de los cauces naturales del agua, la pérdida de vegetación y la erosión del suelo a través de la actividad urbana (Bocco, et. al., 1993; AragónArreola, 1994 y Romo, 1996).

Existe, sin embargo, la posi bilidad de planificar con "Ia naturaleza" en las ciudades. Para ello es preciso no considerar lo natural como algo excepcional y único o como un elemento puramente escénico. AI contrario, el espacio natural debe actuar como receptor de las demás funciones urbanas medianteoperaciones alargo plazo deprotección o restauración, sólo viables a través delacreación deun sistema territorial de espacios naturales. Esto es la creación y mantenimiento de una red de áreas verdes, con distribución por toda la superficie urbana, interconectados entre sí y con el campo circundante. Para ello, deben superarselas propuestas segmentadoras y desarticuladas y reorientarse a una aproximación basada en los procesos naturales y en la problemática aportada por las situaciones de partida del medio a conservar o proteger (Díaz Pineda y Valenzuela, 1989).

En un plano legislativo se sugiere modificar el reglamento, estableciendo un índice deárea verdedeentre 10 y $12 \mathrm{~m}^{2}$ por habitante, que es la norma reconocida internacional mente; y que los fraccionamientos absorban el costo de desalojar del área el exceso de tierra producido por el aplanado (en lugar de arrojarlo a las laderas).

La prevención de desastres se canaliza en el desarrollo urbano a través de la regulación de los usos del suel o que comprende tanto su planeación como su administración. Sin embargo, en el caso deTijuana la limitada concepción que se tiene sobre las áreas verdes, no permite visualizarlas como mecanismos queal mismo tiempo querestablezcan Ios flujos naturales, reduzcan la probabilidad de riesgos, eleven la cal idad visual del paisajeurbano y constituyan un espacio derecreación 
para la población. Esta situación seveagravada por la desarticulación y sectorización que existen en la administración en materia urbana.

Una de las al ternativas más viables para establecer áreas verdes en zonas urbanas situadas dentro de regiones áridas o semiáridos la constituyen las plantas regionales. Su uso reducenotablementeel costo desu mantenimiento y las necesidades deagua, ya que dichas plantas crecerán en un ambiente con características similares o iguales a las de su medio natural. El uso de especies nativas o de especies con pocos requerimientos deagua, por lo tanto, podrían llegar a ser unaalternativa de reforestación viable a mediano plazo. Sin embargo, bajo esta propuesta se debe considerar que la vegetación que habita en ambos lados de la frontera es altamente inflamable.

En la implementación de esta estrategia no se generarían recursos económicos, sin embargo éstos seahorrarían. Primero porqueselograría asegurar, aunque fuese en periodos cíclicos, la recarga de la Presa A belardo L. Rodríguez, lo que disminuirá la necesidad de bombear agua desdeel Río Colorado y el gasto queesto significa, como sucedió en 1996. En segundo lugar, al interior dela ciudad, la forestación urbana reduciría la erosión hídrica ocasionada durantela temporada delluvias, quearrastra grandes cantidades de suel o a las partes bajas dela cuenca y que tan sólo su remoción durantelas inundaciones de 1993 tuvo un costo de 18 millones depesos, además dela pérdida devidas y hogares. ${ }^{6}$

En resumen, los beneficios aportados por mantener una cubierta vegetal, dentro y fuera dela ciudad serían entreotros: la conservación de la biodiversidad, la mitigación de los daños ocasionados por inundaciones y deslaves y la disminución en la probabilidad de que éstos sucedan; el aumento de la calidad de vida debida a la existencia de suficientes áreas verdes y sobre todo la recarga de los mantos acuíferos y por lo tanto una mayor seguridad en el abastecimiento de agua.

${ }^{6}$ Comunicación personal. Sócrates Bastidas, regidor dela Comisión de Desarrollo Urbano y Ecología, Municipio de Tijuana. 
La desmovilización social es producto del desconocimiento delas causas dela degradación ambiental. Es necesario fomentar aún más la participación dela población eincluso incorporar el sector industrial a que también participe. Para ello resulta indispensable crear un flujo continuo deinformación y comunicación quefluya librementeentrela ciudad y sus residentes. La clave radica en facilitar a la población los medios mínimos necesarios para desarrollar su propio potencial de autogestión con prácticas ecológicamente adecuadas, mejorando sus condiciones y calidad de vida. Los individuos y las familias deben aprender sobre estos temas, de forma que puedan tomar decisiones informadas y participar más activamente, en su casa y su colonia.

Resolver el problema de los riesgos existentes dentro de la ciudad deTijuana requiere de una perspectiva integral, en la que seamplíela concepción deárea verdey no seleconsideresolamente como al go de ornato. También serequierela participación detodos desdeel gobierno, la industria, hasta los individuos.

La necesidad de conservar y desarrollar estas áreas puede parecer menos urgente que dedicar estos espacios a la construcción de viviendas, sin embargo debe considerarse que una vez que un área es construida resulta casi imposible (y muy caro) remediar la fal ta deáreas verdes. 


\section{REFERENCIASBIBLIOGRÁFICAS}

Álvarez, M.G. (1996). “Áreas verdes y gestión local”. Tesis deM aestría. Maestría en Administración Integral del Ambiente. El Colegio dela Frontera Norte. México.

XIV A yuntamiento de Tijuana (1995). Plan estratégico deTijuana. México. A ragón-Arreola, M. (1994). "Evaluación de riesgo geológico debido a movimientos de ladera en la ciudad de Tijuana, B.C. México". Tesis de Maestría. CICESE, Ensenada, B.C.

Barradas, V.L. y Seres, J. (1988). "Los pulmones urbanos", Ciencia y D esarrollo. XIII (78):61-72, (enero-febrero). conAcyT. México.

Bazant, T. (1988). M anual de criterios de diseño urbano. Trillas, México. $384 p$.

Bocco, G. Sánchez, R. y Reimann, H. (1993). “Evaluación del impacto de las inundaciones en Tijuana (enero de 1993). Uso integrado de percepción remota y sistemas de información geográfica". Frontera N orte, 5(10): 53-84.

Colegio deA rquitectos deM exicali A.C. (1985). Leyes y reglamentos que rigen el desarrollo urbano del Estado de Baja California. Colegio de A rquitectos deM exicali A.C. México.

Díaz Pineda, F. y M. Valenzuela (1989). Los espacios naturales en áreas urbanasy periurbanas. En: Casa deVelázquez (Ed.): Supervivencia de los Espacios $N$ aturales. M o. A gricultura, Pesca y Alimentación. Madrid: 335-347.

García, E. y Mosiño, P.A. (1968). Los climas de Baja California. Comité Nacional M exicano para el Decenio Hidrológico. Internacional. Memorias 1966-1967. Instituto deGeofísica. UNAM, M éxico.

García, R. (1988). Estratigrafía y propiedades de los suel os de Tijuana,

B. C.XIV Reunión M exicana de Mecánica deSuelos. Monterrey, N.L. México.

INEGI (Instituto Nacional de Estadística, Geografía e Informática) (1990). X Censo General de Población y V ivienda. M éxico.

Forman, R.T.T. (1995). Land M osaics. The Ecology of landscapes and regions. CambridgeUniversity Press. Cambridge.

Gilbert, O.L. (1989). The E cology of U rban H abitats. Chapman and Hall. London. 
Minnich, R.A . (1983). “FireM osaics in Southern California and Northern Baja Cal ifornia". Science, 219:1287-1294.

Minnich, R.A. y Bahre, C. J. (1996). "Wildland Fire and Chaparral Succesion A long the Cal ifornia-Baja Cal ifornia Boundary". Int. J. W ildl andfire, 5(1):13-24.

Minnich, R.A. y Franco, E. (1998). Land of Chamise and Pines. H istorical A ccounts and Current Status of $N$ orthern Baja California's V egetation. University of California Press. Botany Volume 80.

O'Leary, J. (1989). “California Coastal Sage Scrub: General

Characteristics and Considerations for Biological Conservation". En: Schoenherr (Editors), D angered Plant Communities of Southern California. Proceedings of the15th A nnual Symposium. Southern California Botanists. Special Publication, núm. 3. pp. 24-41.

Oberbauer, T. (1986). "Environmental Planning in the Otay Border Area”. En: Álvarez, J. y Castillo, V.M. (Coordinators), Ecología y Frontera. Universidad A utónoma deBaja Cal ifornia. pp. 108-113. Romo, Ma. L. (1996). "Riesgos naturales y vulnerabilidad social en la zona urbana de Tijuana". Tesis de Maestría. Maestría en Administración Integral del A mbiente. El Colegio dela Frontera Norte. 124p.

Rzedowski, J. (1978). V egetación de M éxico. Editorial Limusa, México. Smith, K. (1992). Environmetal H azards. A ssesing Risk \& R educing D isaster. Routledge. London.

Wiggins, I. (1980). Flora of Baja California. Stanford University Press. Stanford, California.

Wolch, J. (1996). "Zoöpolis". Capital ism, N atureand Social ism. 7(2): 21-47. 
LA REFORESTACIÓN DE TIJUANA 